\title{
Fatigue testing of a proximal femoral hip component
}

\author{
W Macdonald ${ }^{1 *}$, L V Carlsson ${ }^{1,2}$, N Gathercole ${ }^{3}$ and C M Jacobsson ${ }^{1}$ \\ ${ }^{1}$ Department of Biomaterials Research, Institute for Surgical Sciences, University of Gothenburg, Sweden \\ ${ }^{2}$ Department of Orthopaedics, Sahlgrens University Hospital, University of Gothenburg, Sweden \\ ${ }^{3}$ SMS Consultants, Materials Research Centre, Department of Engineering and Applied Science, University of Bath, \\ UK
}

\begin{abstract}
The Gothenburg Osseointegrated Titanium (GOT) implant is a novel total hip replacement including a metaphyseal loading proximal femoral component fixed in the retained femoral neck. Endurance testing was performed under conditions analogous to ISO 7206-4: 1989. The cementfree implant is not fixed distally within the intramedullary canal, so distal embedding (as specified in the standard) would have been unrealistic. Instead glass-fibre-reinforced epoxy (GFRE) bushings were used to model reduced bone support mid-length at the medial cortex and distally at the lateral cortex. Such support simulated proximal bone loss, realistically reproducing the effect of osteolysis or fixation failure. Under such conditions the component survived unbroken for 10 million cycles at $3.0 \mathrm{kN}$ peak load.
\end{abstract}

Keywords: hip replacement, fatigue testing, femoral component, GFRE bushings, GOT implant

\section{NOTATION}

cpTi commercially pure titanium

GFRE glass-fibre-reinforced epoxy

$\mathrm{GPa} \quad$ gigapascals $=10^{9} \mathrm{~N} / \mathrm{m}^{2}$

ISO International Standards Organization

$\mathrm{kN} \quad$ kilonewtons

PE polyethylene

$Q$-angle angle between the axis of the femoral shaft and the mechanical axis of the lower limb in normal stance (which is defined by a line between the femoral head and the centre of the knee)

$R$ ratio ratio between the maximum and minimum loads applied during cyclic testing $=F_{\min } / F_{\max }$

\section{INTRODUCTION}

The purpose of pre-clinical testing is to ensure the suitability of implant components for the proposed service conditions and their ability to withstand those service conditions without catastrophic failure. In practice, this requires two forms of testing: laboratory or computer

The MS was received on 8 October 2002 and was accepted after revision for publication on 2 January 2003.

* Corresponding author: 5 St James' Square, Boscombe, Dorset BH5 $2 B X, U K$. simulated testing under normal conditions for the design lifetime to ensure satisfactory performance under normal conditions and laboratory or simulated testing under extreme or abnormal conditions to prevent or predict disastrous failure modes.

Fatigue failure of hip components was first recognized in Charnley's pioneering series [1]. The incidence of fracture in clinical series was then reported to assume values varying from 0.23 to 11 per cent [1-5].

The causes of fracture were thought to be calcar resorption [1], proximal loosening [6] or proximal loosening with overstress [7] plus an element of torsion and bending [8]. However, failure of some components was observed in the absence of loosening or cement inadequacy [9], and failure was also observed in 'highstrength' components [8] and with third-generation cement and stems [10]. Despite finding various metallurgical defects, Rostoker and colleagues [11] and Collis [4] concluded that these were not the direct cause of fracture, which was rather due to conditions causing excessive stress (heavy patient, loosening and calcar loss) unfortunately coinciding with stem weakness or defects. Although some concluded that 'fracture of the metal is merely the ultimate fate of a loosened prosthesis' (reference [2], p. 654), Ducheyne and colleagues observed [5] that some patients seemed able to apply sufficiently severe loading conditions to even well-fixed cemented components. This confusion of causes left problems for the designers of pre-clinical tests.

In fact, the consequences of fatigue failure (the need 
to excavate cement and well-fixed distal fragments) dictated the mode of fatigue testing [12], more than the actual consideration of clinical failure. Callaghan et al. observed [13] that prediction of imminent failure would enable removal of an intact component before failure (but after loosening), which was preferable to retrieving the distal fragment from deep within the femoral medullary canal.

For this reason, the first standard fatigue test defined support conditions modelling proximal loosening but firm fixation distally [14]. Subsequent observations of the contribution of torsion and bending to failure led to a further test procedure with similar support conditions but more rigorous loadings to mimic the out-of-plane bending of normal gait inducing increased stress in curved intramedullary stems $[\mathbf{1 2}, \mathbf{1 5}]$. Endurance limits were set to model ten years' clinical service under normal gait, or 5 million cycles under $3 \mathrm{kN}$ sinusoidal load [16], but when laboratory failures of clinically successful implants occurred at these loads under the conditions of ISO 7206-4 [17], an alternative endurance limit of $2 \mathrm{kN}$ was proposed for the torsional set-up $[\mathbf{1 2}, \mathbf{1 8}-\mathbf{2 0}]$.

Despite these precautions, even the strongest designs of femoral component have exhibited unexpected premature failure under unusual clinical circumstances $[\mathbf{1 0}, \mathbf{2 1}]$. Perhaps Ducheyne and colleagues were rightthat some patients will apply excessive loads to any component [5].

The standard tests were developed for conventional intramedullary implants, as their titles imply. Tests for unconventional designs of implants have tended to follow alternative strategies, often testing under optimal support conditions rather than failure modes [22, 23] or even preferring computer modelling to physical testing [24-26].

Endurance testing of implants has historically been driven by the failure modes experienced in clinical practice. For pre-clinical testing of novel implants, a hierarchy of needs can thus be postulated. Firstly, endurance testing is required to ensure that the design can withstand the rigours of normal physiological function. Then, the patterns of clinical failure need to be understood and further endurance testing should ensure that the worst form of clinical failure, or the least recoverable surgically, is avoided.

\section{OSSEOINTEGRATED IMPLANTS}

The breakthough provided by Charnley was the combination of an intramedullary stem with cemented fixation, a small head and a polyethylene (PE) socket [27]. The intramedullary stem inserted down the femoral shaft provided longer term stability regardless of the boneimplant interface, and the acrylic cement provided immediate secure interface stability. However, the pre- dominant failure mode was osteolysis and loosening at the endosteal interfaces.

Osteolysis was shown to be avoidable by following the principles of osseointegration, first in dental and maxillofacial applications [28-30] and subsequently in orthopaedics $[31,32]$. With interfacial stability attainable immediately and in the long term without requiring distal intramedullary fixation, the form and design of the implants could be selected to minimize stressshielding and micromotion [33], specifically by restricting the implant to the proximal femur. The resulting design was provisionally named the Gothenburg Osseointegrated Titanium hip (GOT hip) (Fig. 1). With such geometry, the standard endurance test conformation was no longer appropriate.

In particular, since the implant was not inserted or fixed distally within the medullary canal, embedding of the distal portion of the implant with cantilevered exposure of the proximal half to two-thirds of the implant (the fixation specified for the ISO test) was unrepresentative. Instead, implant/bone support is achieved within

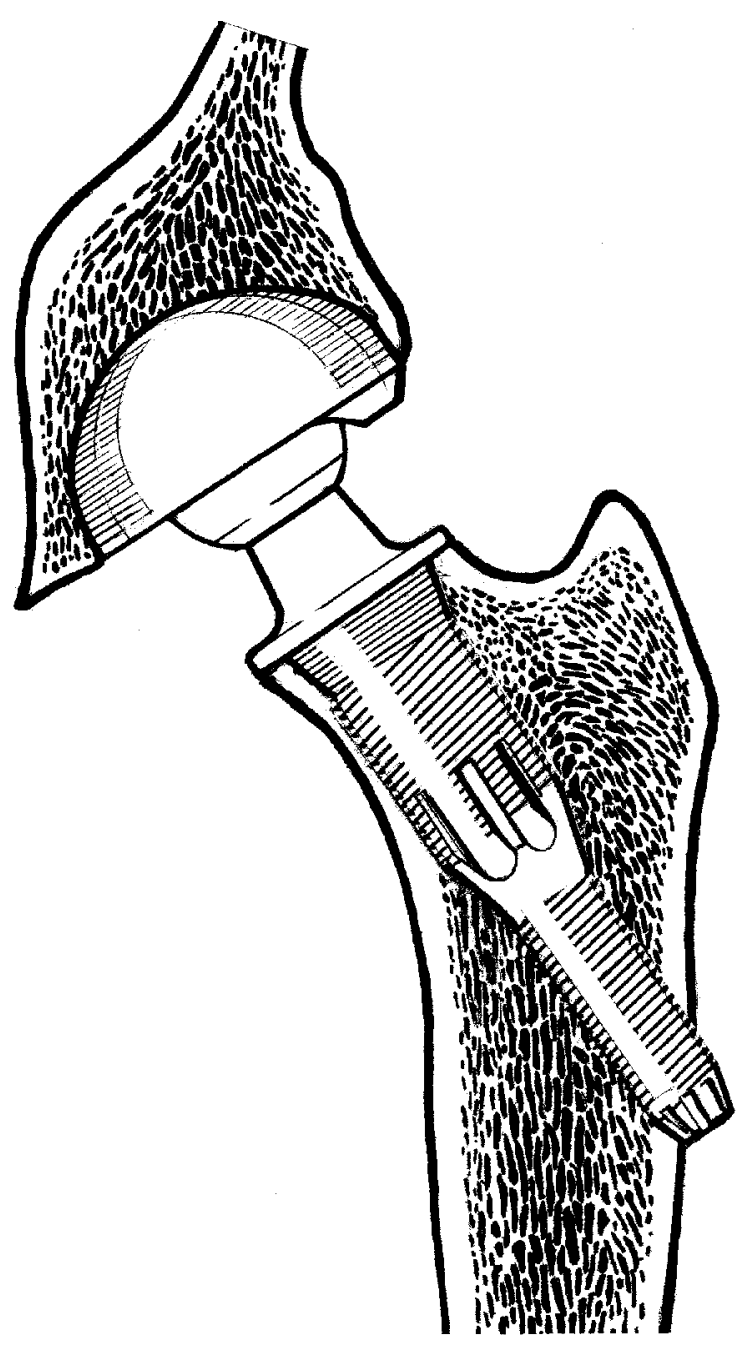

Fig. 1 Schematic of the GOT component implanted in the femoral neck and the lateral cortex 
the femoral neck and at the lateral cortex [32] (Fig. 1). It was reasoned that generalized bone loss would allow the component to sink into varus, whereas specific periprosthetic osteolysis would reduce the total length of bone support in the femoral neck, while retaining some medial calcar support. Initial clinical trials confirmed this conclusion [32].

Failure mode testing required support in the same manner, with distal support modelling the lateral cortex and some reduced support along the femoral neck region (Fig. 2). Under these conditions, endurance testing should ascertain the ability of the implant to endure the functional loads of normal activities.

As a further safety measure, using proximal fixation only and a rotationally symmetrical form of implant means that revision is much easier. The consequences of implant failure are not as catastrophic as with well-fixed distal intramedullary implants.

Undertaking tests indicated by the two-level hierarchy of needs described in the previous section suggested a second major test form. Normal service testing would further require support of the implant at the lateral cortex and throughout the proximal or femoral neck region, with application of the most severe loads to be anticipated in clinical practice in order to determine the ability of the implant to sustain the loads thus applied and the ability of the adjacent bone to endure the conditions also [32].

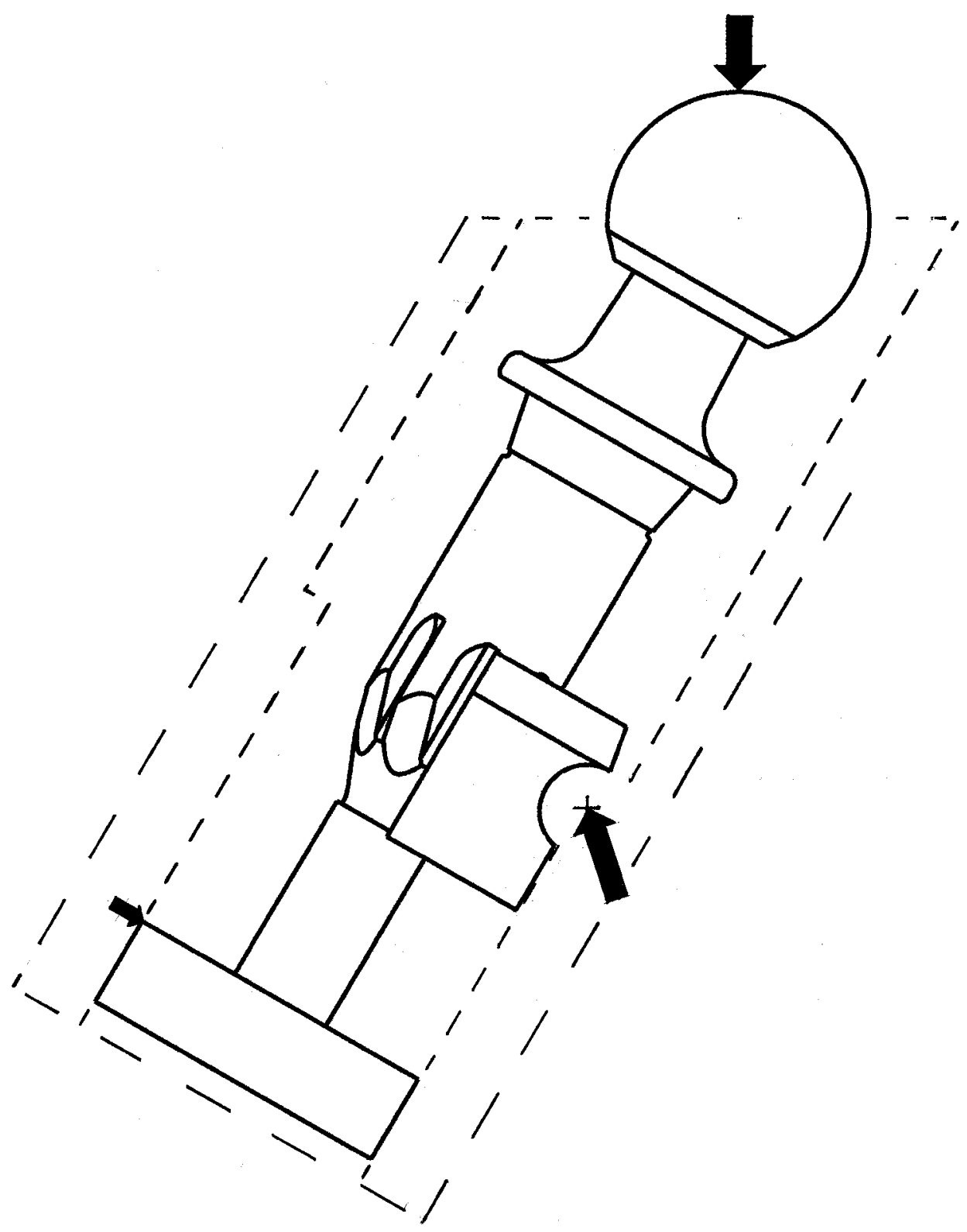

Fig. 2 The fatigue test conformation, showing reduced and offset proximal contact and separate distal support (modelling the lateral cortex). The body of the test rig, represented by the dashed line, enclosed the component and bushes and enabled wet testing 


\section{MATERIALS AND METHODS}

Bone support was modelled using bushings of glassfibre-reinforced epoxy (GFRE) material representative of cortical bone (grade 10G40, elastic modulus 12.7 GPa; Tufnol Limited, Birmingham) supported within a stainless steel housing. The use of an enclosed housing enabled testing in simulated physiological fluids if required. The GFRE bushes were machined to net shape, with the surfaces interfaced to the implant being finished by the self-tapping feature of the implant. Two GFRE bushes were employed (Figs 2 and 3), one supporting the distal tip of the implant (modelling the lateral femoral cortex), fitting flush within the housing, and a second fitted more proximally to the implant, with a gap immediately beneath the collar or femoral neck osteotomy site, supported by a transverse pivot. The

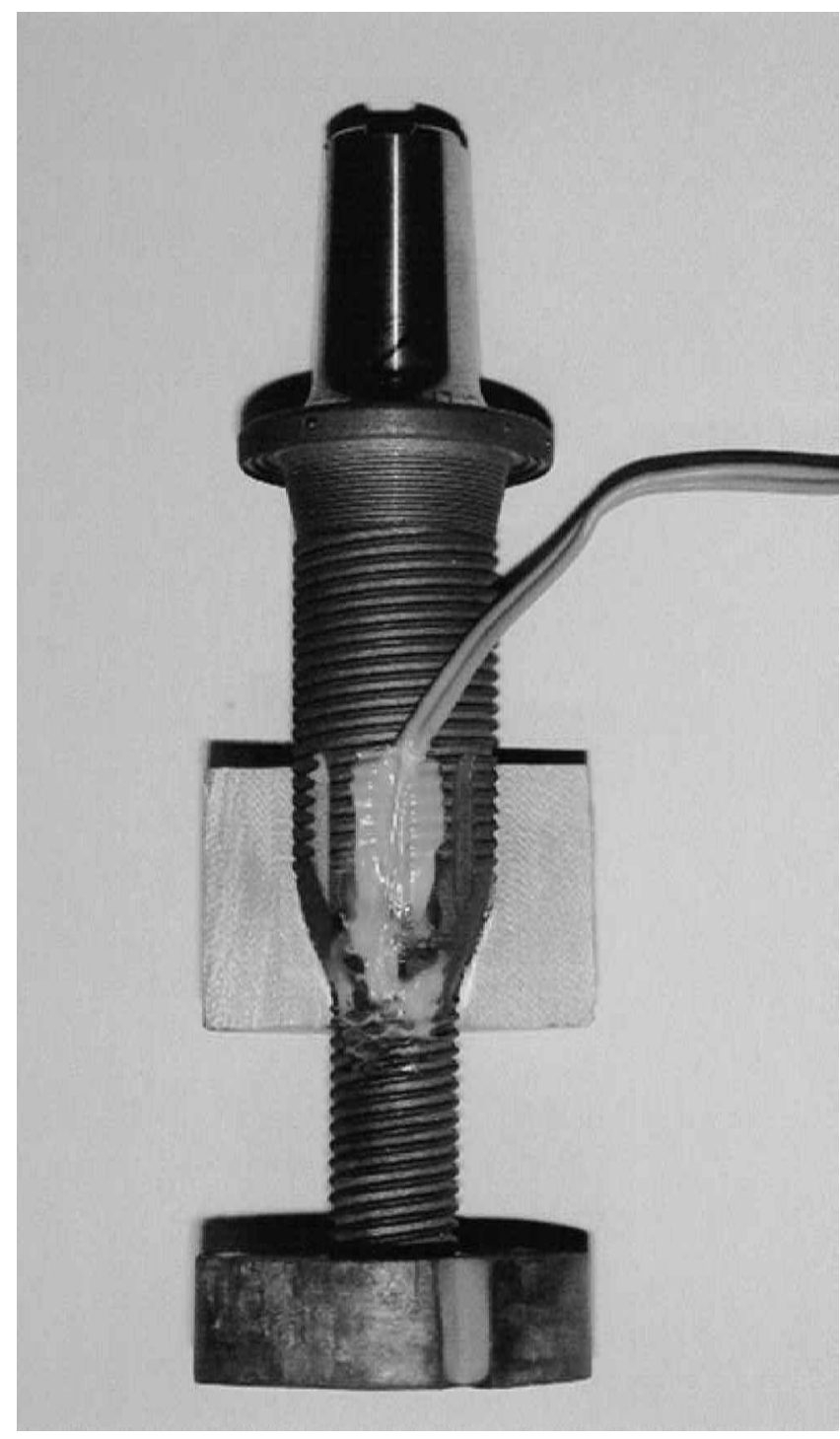

Fig. 3 Test component and two GFRE bushes in place (this component was strain-gauged to validate finite element analysis) second bush models support but not firm fixation at the medial cortex. The implant is designed to contact the endosteal surfaces of the femoral neck over a length of $40 \mathrm{~mm}$; this was reduced to the distal $10 \mathrm{~mm}$ for the endurance tests, modelling $30 \mathrm{~mm}$ of lost implant/bone contact.

The Gothenburg osseointegrated titanium (GOT) femoral component is clinically inserted within the femoral neck, with an average implant/shaft angle of $149^{\circ}$ [33]. The test geometry defined by the more rigorous ISO standard [15] requires $10^{\circ}$ adduction and $9^{\circ}$ hip flexion combined with a vertical test load; this level of adduction places the stem in 'neutral' or at the $Q$-angle, the normal mechanical functional position [33]. The 'with torsion' geometry of ISO 7206-4: 1989(E) defines the worst mode loading for stemmed intramedullary implants, for which the added flexion angle induces an 'out-of-plane' torsional stress component in the implant. For a rotationally symmetrical component such as the GOT femoral, there is no torsional effect - the added flexion simply increases the effective transverse component of the resultant force. Geometrically, the sum of the two angles is $13^{\circ} 25^{\prime}$, which results in a component/ vertical inclination of $162^{\circ} 25^{\prime}$, or $17 \frac{1}{2}^{\circ}$. However, the component inclination in the present tests was increased to $30^{\circ}$, to represent a 'worst case' scenario.

Test femoral components of the GOT hip were obtained ready for sterilization. The components were produced from commercially pure titanium (cpTi) grade IV, ceramic bead blasted and cleaned after finishing and (in the final test series) surface treated for improved bone reaction. Once the component had been fitted into the GFRE bush, the implant/bush combination was inserted into the fatigue housing to the appropriate level and the pivot groove was milled through the proximal bushing using an end mill. A zirconia ceramic head of $28 \mathrm{~mm}$ diameter and 'medium' neck extension was fitted to the stem's taper trunnion, following the manufacturer's guidelines. The fatigue housing was mounted firmly on the actuator of the servo-hydraulic test machine (Model 1332, Instron Limited, High Wycombe) (Fig. 4 depicts the two housings mounted in parallel; that on the right was mounted alone centrally on the actuator for single component tests).

The vertical load was applied through a purpose-built steel loading nose mounted on a horizontal linear bearing to accommodate medio-lateral motion of the ceramic head due to bending of the implant. The linear bearing was attached to the load cell of the Instron machine, with a 5 or $25 \mathrm{kN}$ load cell dependent on the test load applied. Loads were applied at an $R$ ratio of 10 (the minimum load is 10 per cent of the chosen maximum load) and at a maximum frequency of $2.6 \mathrm{~Hz}$ (depending on the total actuator motion required). The test force and crosshead displacement were continuously 


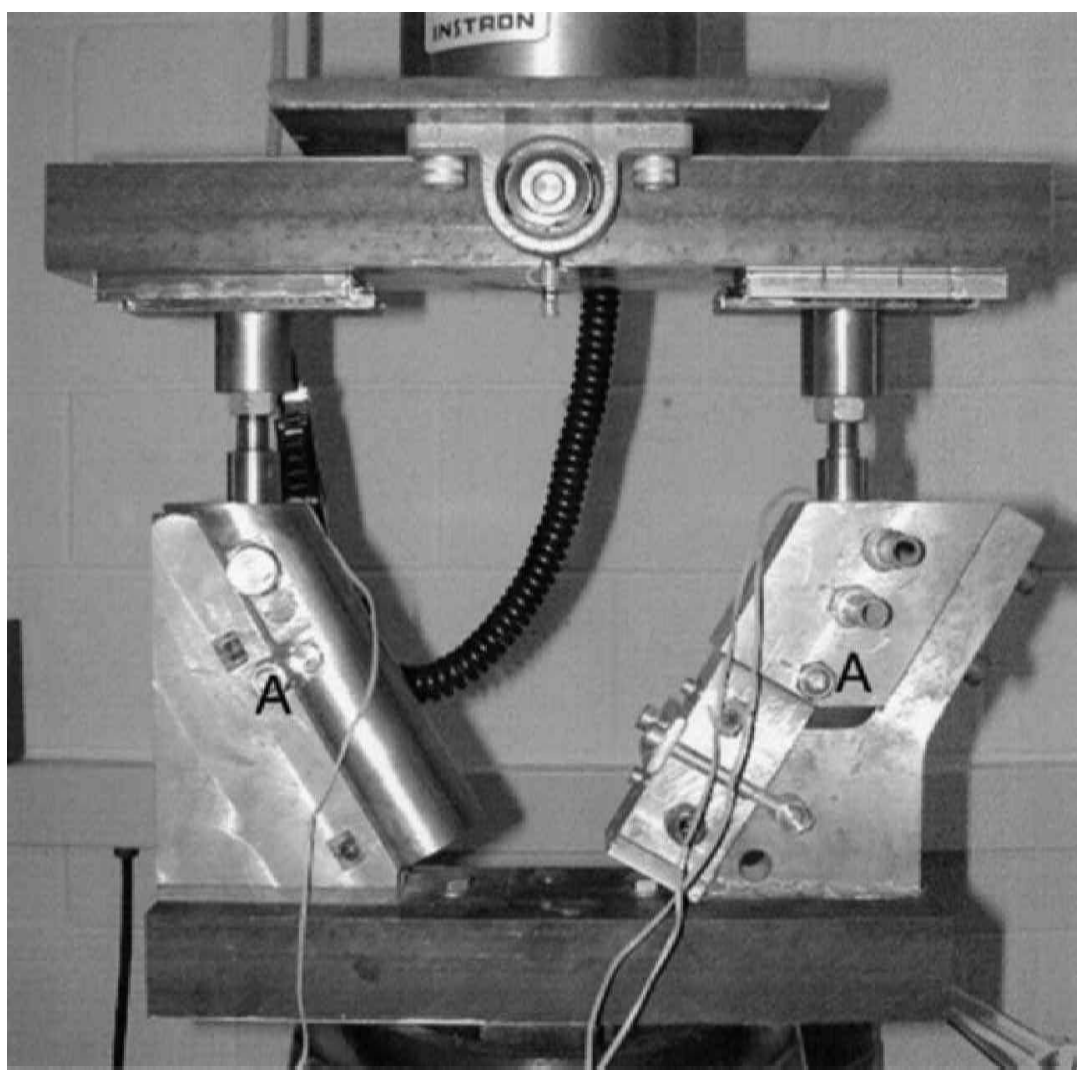

Fig. 4 Tandem fatigue rig in the Instron machine. The pivot bolts for the proximal bushing (marked A) can be seen

monitored, the test being automatically halted when displacement increased significantly beyond the initial observed range.

For clinical use the implant components are surface treated to enhance bone reaction, which might allow chemical interaction or attack within the bodily environment. Therefore, final testing of the components was undertaken immersed in 3\% Ringer's solution and no faster than $3 \mathrm{~Hz}$. The test solution was stored in a tank beside the test machine and continuously pumped to the test housing by a submerged pump, the temperature being controlled so that the fatigue housing was maintained at $37^{\circ} \mathrm{C}$.

For low-load-high-cycle testing, with the frequency restriction due to the surface treatment, a second fatigue housing and loading nose were prepared, and two specimens were tested in parallel (Fig. 4). In this way the machine time for the test programme was halved for these tests.

\section{RESULTS}

All components tested at $3 \mathrm{kN}$ survived to 10 million cycles, at which point the tests were stopped and the specimens were removed from the rig. All the remaining specimens were examined after failure, revealing cracking at the narrowest section, as expected. Location of the crack initiation site seemed to be related to the position of the thread runout next to the narrowest section, and this was more thoroughly examined. Under low-load/high-cycle tests there was a weak correlation between thread position and endurance, but not consistently, and at higher loads no relationship was found. Overall, therefore, it was concluded that neither thread position nor implant alignment had a strong influence on fatigue lifetime.

With all the results plotted on an S-N (stressendurance) curve, it can be seen that the GOT implant tested under the conditions described (Fig. 5) shows an endurance limit of $3.0 \mathrm{kN}$. Some spread of the results was observed over the lower load ranges, and therefore an $\mathrm{S}-\mathrm{N}$ curve of the mean lifetimes at each load level was also plotted (Fig. 6).

Inspection of failed components revealed classic fatigue failure signs; concentric curved lines called 'beach' or 'river' markings denoting cyclic crackfront advance. Testing was terminated before total component fracture could occur, so this was achieved by massive overload. Although there were some minor signs of staining of early crack segments in the longer lasting specimens, there was no evidence of corrosion involvement in crack initiation or propagation. 


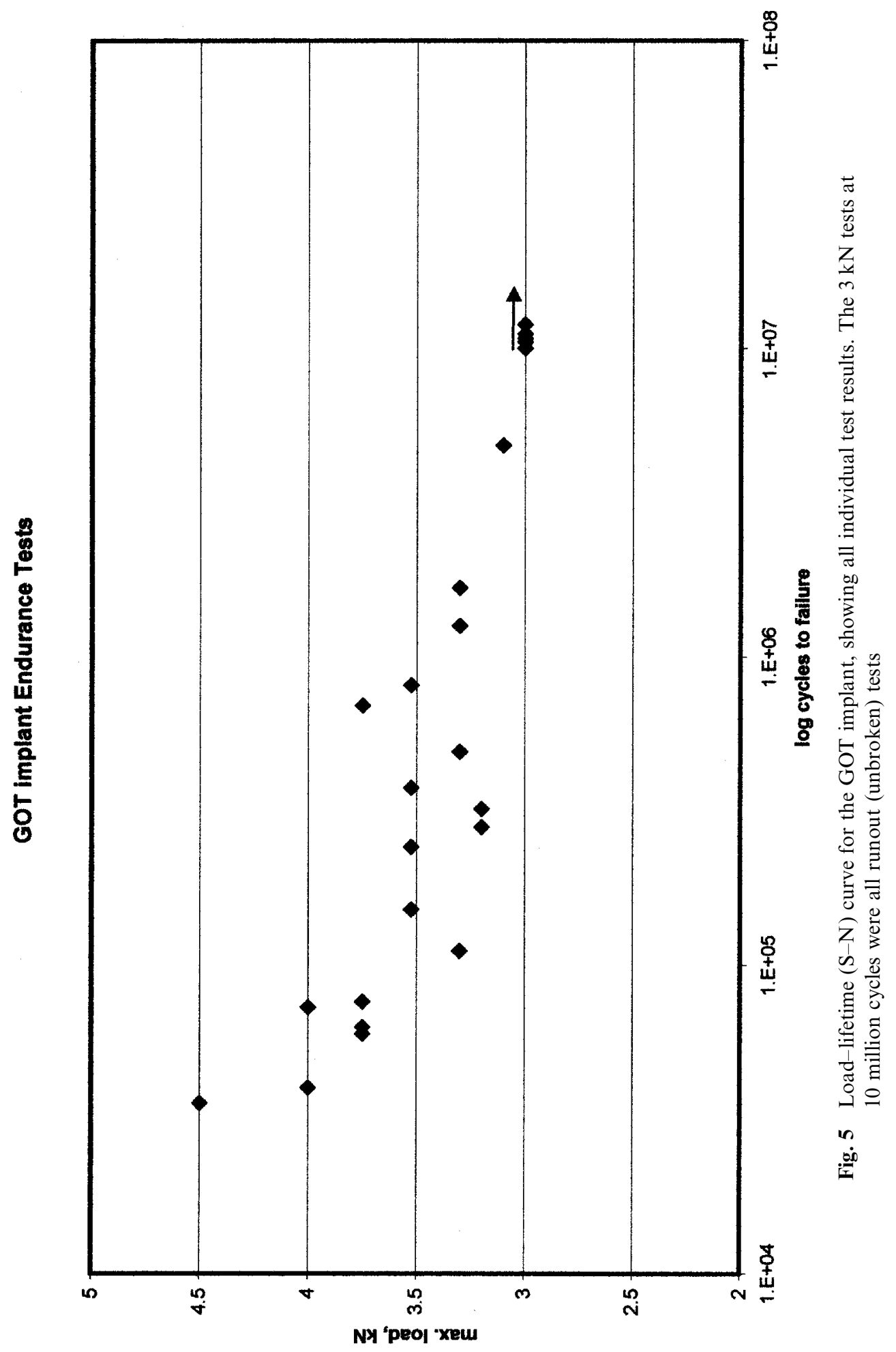




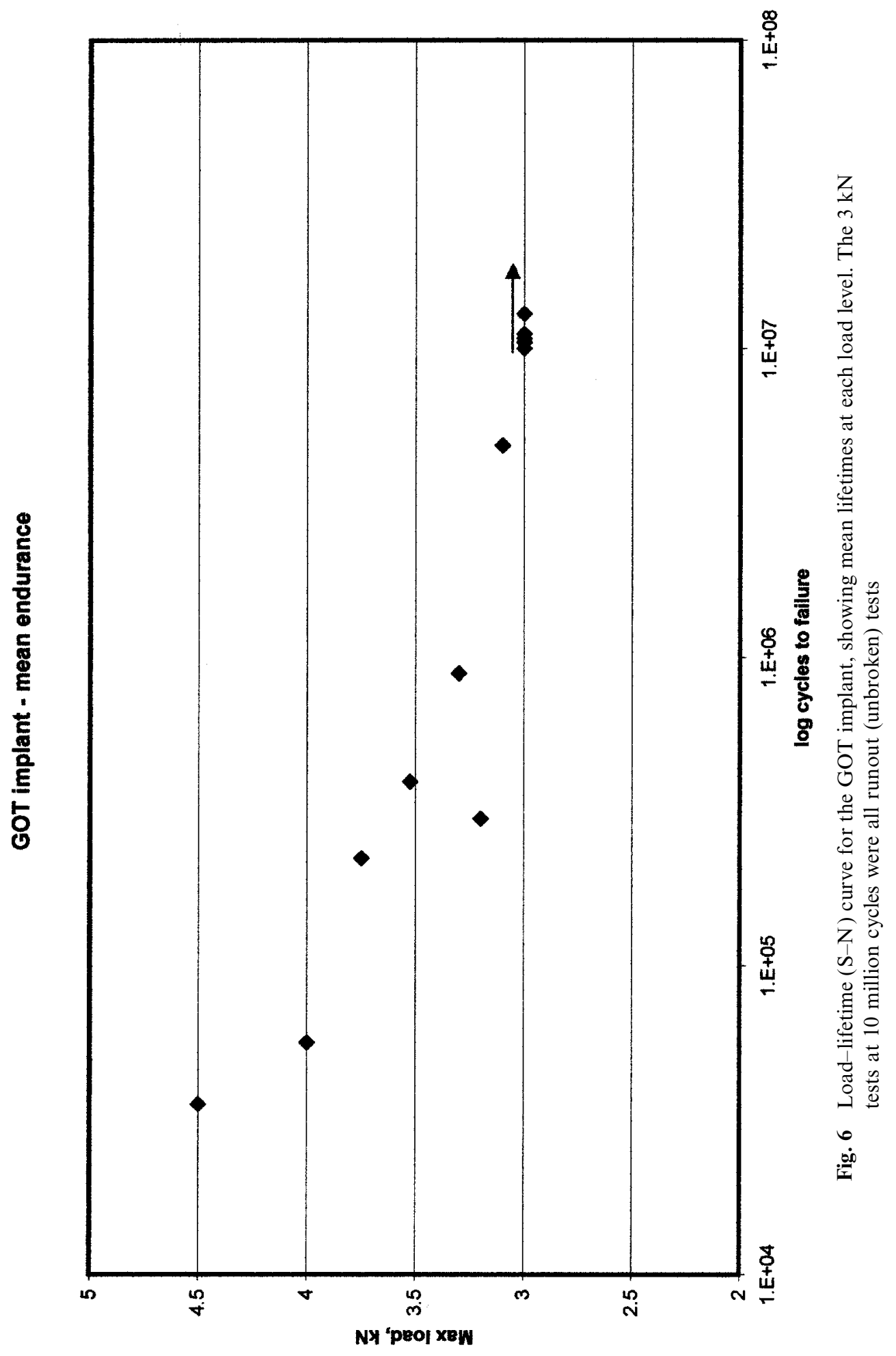




\section{DISCUSSION}

Describing the development of the standard test methods for conventional stemmed intramedullary implants, Professor Paul wrote (reference [34], p. 389):

... the fracture of the stem of the femoral component was a secondary failure after the failure of the fixation of the proximal part of the implant. It was felt desirable that in these circumstances the implant should not fail since removal of the distal part of the implant which would generally be firmly fixed would be a difficult procedure. The preferred clinical history then would be that the implant became so loose as to develop pain for the patient on a level which would require surgical treatment and the intact implant could then be removed.

Paul also stated that:

Implants have been developed of composite construction resulting in flexibility in bending more closely matching that of the proximal femur with the objective of reducing bone resorption due to 'stress shielding'. No mechanical tests have yet been specified for these.

Having developed a novel proximal femoral implant such as the GOT implant, it was necessary to develop appropriate endurance tests, in the absence of tests specified by the Regulatory Bodies. The $\mathrm{S}-\mathrm{N}$ curve from this series of tests shows that fracture has been achieved at elevated loads, demonstrating fatigue behaviour typical of most metals and especially titanium, with an endurance limit in the range of $10^{5}-10^{6}$ cycles [35]. This behaviour gives confidence that the long-term survival of the implant under such conditions can be predicted by these tests.

The ISO Standard tests for intramedullary stems were given more rigorous geometry during their development, but at reduced load magnitude, because the additional torsional component induced failure in implants that had not failed clinically [17]. For testing under these conditions, the specified minimum endurance performance is now a cyclic load of $2.0 \mathrm{kN}$ on a minimum load of $0.3 \mathrm{kN}[\mathbf{2 0}]$. For a proximal implant with rotational symmetry like the GOT component, loaded through a spherical head bearing, the 'more rigorous' geometry does not induce any out-of-plane components, and so the geometry described (Fig. 2) is still appropriate, at the specified limit of $2.3 \mathrm{kN}$ maximum load and 5 million cycles. Five specimens having survived 10 million cycles at $3.0 \mathrm{kN}$, it can be concluded that the GOT implant has exceeded the performance requirements of ISO 7206-8: 1995(E).

Distal embedding was not appropriate to this implant form due to the fixation mechanics in the body. For stemmed implant tests it has been shown that varying the embedding medium can also alter the observed endurance behaviour [36]. Modelling embedded geometry is also more complex with finite element (FE) analysis, whereas using a GFRE bush of modulus similar to cortical bone with the simplified geometry of a transverse pivot enabled clearer FE modelling. It is suggested that realism is not reduced by this approach, while computational verification is made easier.

In fact, tests of a prototype of the implant under the same support conditions produced fracture in some components at $2.6 \mathrm{kN}$, with crack initiation similar to that observed in the present tests. A pilot clinical trial of the prototype had been undertaken, in which one component fractured in an active, heavy $(105 \mathrm{~kg})$ patient who exhibited excessive proximal bone loss [37]. It is therefore concluded that this mode of testing realistically models the clinical situation.

As expected with titanium, which is known to be 'notch sensitive' [35], crack initiation appeared to be affected by the stress concentration of a dramatic change in section, but not so clearly by thread runout. Due to the implant design and heavy surface blasting, the general location of crack initiation was predictable but the effect of the threaded feature did not appear to lead to systematic weakening of the implant.

While the international standards specify conditions for conventional stemmed intramedullary devices, unconventional devices require alternative and more appropriate test conditions. The use of GFRE bushings models the clinical situation of bone support as effectively as embedding resins, while obviating the variability introduced into the ISO tests by resin properties [36].

With a combination of all the conditions described it has been possible to test realistically the endurance properties of the implant in the laboratory and to demonstrate adequate long-term strength.

\section{CONCLUSIONS}

1. The GOT femoral component has been endurance tested under conditions analogous to ISO 7206-4: 1989(E) and demonstrated an endurance limit at 10 million cycles of $3 \mathrm{kN}$, which exceeds the $2 \mathrm{kN}$ threshold of ISO 7206-8.

2. Test conditions appropriate to this form of implant and realistic to clinical performance have been developed.

\section{REFERENCES}

1 Charnley, J. Fracture of femoral prostheses in total hip replacement: a clinical study. Clin. Orthop., 1975, 111, 105-120.

2 Carlsson, Å. S., Gentz, C.-F. and Stenport, J. Fracture of the femoral prosthesis in total hip replacement according to Charnley. Acta Orthop. Scand., 1977, 48, 650-655.

3 Wroblewski, B. M. Fractured stem in total hip replacement. Acta Orthop. Scand., 1982, 53, 279-284.

4 Collis, D. K. Femoral stem failure in total hip replacement. J. Bone Jt Surg., 1977, 59-A(8), 1033-1041. 
5 Ducheyne, P., De Meester, P., Aernoudt, E., Martens, M. and Mulier, J. C. Fatigue fractures of the femoral component of Charnley and Charnley-Müller type total hip prostheses. J. Biomed. Mater. Res. Symp., 1975, 6, 199-219.

6 Gruen, T. A., McNiece, G. M. and Amstutz, H. C. 'Modes of failure' of cemented stem type femoral components. Clin. Orthop., 1979, 141, 17-27.

7 Galante, J. O. Causes of fractures of the femoral component in total hip replacement. J. Bone Jt Surg., 1980, 62-A(4), 670-673.

8 Miller, E. H., Shastri, R. and Shih, C.-I. Fracture failure of a forged vitallium prosthesis. J. Bone Jt Surg., 1982, 64-A(9), 1359-1363.

9 Chao, E. Y. S. and Coventry, M. B. Fracture of the femoral component after total hip replacement. J. Bone Jt Surg., 1981, 63-A(7), 1078-1094.

10 Woolson, S. T., Milbauer, J. P., Bobyn, J. D., Yue, S. and Maloney, W. J. Fatigue fracture of a forged cobaltchromium-molybdenum femoral component inserted with cement. J. Bone Jt Surg., 1997, 79-A(12), 1842-1848.

11 Rostoker, W., Chao, E. Y. S. and Galante, J. O. Defects in failed stems of hip prostheses. J. Biomed. Mater. Res., 1978, 12, 635-651.

12 Paul, J. P. Development of standards for orthopaedic implants. Proc. Instn Mech. Engrs, Part H: J. Engineering in Medicine, 1997, 211(H1), 119-126.

13 Callaghan, J. J., Pellicci, P. M., Salvati, E. A., Garvin, K. L. and Wilson, P. D. J. Fracture of the femoral component analysis of failure and long-term follow-up of revision. Orthop. Clin. N. Amer., 1988, 19(3), 637-647.

14 ISO 7206-3: 1988 Determination of Endurance Properties of Stemmed Femoral Components without Application of Torsion, 1988 (International Organization for Standardization).

15 ISO 7206-4: 1989 Determination of Endurance Properties of Stemmed Femoral Components without Application of Torsion, 1989 (International Organization for Standardization).

16 ISO 7206-7: 1993 Endurance Performance of Stemmed Femoral Components without Application of Torsion, 1993 (International Organization for Standardization).

17 Humphreys, P. K., Orr, J. F. and Bahrani, A. S. Testing of total hip replacements: endurance tests and stress measurements. Part 1: endurance tests. Proc. Instn Mech. Engrs, Part H: J. Engineering in Medicine, 1990, 204(H1), 29-34.

18 Humphreys, P. K., Orr, J. F. and Bahrani, A. S. Testing of total hip replacements: endurance tests and stress measurements. Part 2: stress measurements. Proc. Instn Mech. Engrs, Part H: J. Engineering in Medicine, 1990, 204(H1), 35-41.

19 Paul, J. P. Comment on: Bending and fracture of the femoral component in cemented total hip replacement. J. Biomed. Engng Lett., September 1992, 14, 444.

20 ISO 7206-8: 1995 Endurance Performance of Stemmed Femoral Components with Application of Torsion, 1995 (International Organization for Standardization).

21 Lee, E. W. and Kim, H. T. Early fatigue failures of cemented, forged, cobalt-chromium femoral stems at the neck-shoulder junction. J. Arthroplasty, 2001, 16(2), 236-238.

22 Postak, P. D., Polando, G., Pugh, J. W. and Greenwald,
A. S. A new method of fatigue testing for proximally supported femoral stems. In 57th Annual Meeting of the American Academy of Orthopaedic Surgeons, New Orleans, Louisianna, 1990 (American Academy of Orthopaedic Surgeons).

23 Krygier, J. J., Dujovne, A. R. and Bobyn, J. D. Fatigue behavior of a titanium femoral hip prosthesis with proximal sleeve-stem modularity. J. Appl. Biomater., 1994, 5, 195-201.

24 Taylor, D., Martin, C., Cornelis, B. and Jones, M. E. B. An isoelastic prosthesis using a new composite material. Proc. Instn Mech. Engrs, Part H: J. Engineering in Medicine, 1993, 209(H2), 121-125.

25 Akay, M. and Aslan, N. An estimation of fatigue life for a carbon fibre/poly ether ether ketone hip joint prosthesis. Proc. Instn Mech. Engrs, Part H: J. Engineering in Medicine, 1995, 209(H2), 93-103.

26 Simões, J. A. O., Taylor, M., Marques, A. T. and Jeronimidis, G. Preliminary investigation of a novel controlled stiffness proximal femoral prosthesis. Proc. Instn Mech. Engrs, Part H: J. Engineering in Medicine, 1998, 212(H3), 165-175.

27 Charnley, J. The bonding of prosthesis to bone by cement. J. Bone Jt Surg., 1964, 46-B(3), 518-529.

28 Albrektsson, T., Brånemark, P.-I., Hansson, H.-A. and Lindström, J. Osseointegrated titanium implants. Requirements for ensuring a long-lasting, direct bone-toimplant anchorage in man. Acta Orthop. Scand., 1981, 52, 155-170.

29 Albrektsson, T. and Albrektsson, B. Osseointegration of bone implants. Acta Orthop. Scand., 1987, 58, 567-577.

30 Albrektsson, T. and Jacobsson, M. Bone metal interface in osseointegration. J. Prosthet. Dent., 1987, 57(5), 597-607.

31 Carlsson, L. V. On the development of a new concept for orthopaedic implant fixation. PhD thesis, Department of Handicap Research and Department of Orthopaedics, Göteborgs Universitet, Gothenburg, Sweden, 1989.

32 Albrektsson, T., Carlsson, L. V., Jacobsson, M. and Macdonald, W. Gothenburg osseointegrated hip arthroplasty. Clin. Orthop. Related Res., 1998, 352, 81-94.

33 Macdonald, W., Carlsson, L. V., Jacobsson, M. and Lee, T. Q. A proximal femoral implant preserves physiological bone deformation: a biomechanical investigation in cadaveric bones. Proc. Instn Mech. Engrs, Part $H$ : J. Engineering in Medicine, 2003, 217(H1), 41-48.

34 Paul, J. P. Strength requirements for internal and external prostheses. J. Biomechanics, 1999, 32, 381-393.

35 Polmear, I. J. Light alloys. In Metallurgy and Materials Science, 3rd edition (Eds R. W. K. Honeycombe and P. Hancock), 1989, p. 354 (Edward Arnold, London).

36 Viceconti, M., Toni, A. and Giunti, A. Influence of the embedding media on the results of standard in vitro fatigue tests on the femoral components. Proc. Instn Mech. Engrs, Part H: J. Engineering in Medicine, 1994, 208(H3), 131-137.

37 Carlsson, L. V., Albrektsson, B. E. J., Jacobsson, M., Macdonald, W., Regnér, L. and Weidenhielm, L. A prospective randomized clinical trial of the Gothenburg osseointegrated titanium hip arthroplasty. J. Arthroplasty (submitted). 